\title{
Thin, flexible, capacitive force sensors based on anisotropy in 3D-printed structures
}

\author{
Gerjan Wolterink $^{1,2}$, Remco Sanders ${ }^{1}$, Gijs Krijnen ${ }^{1}$ \\ ${ }^{1}$ Robotics And Mechatronics group, University of Twente, Enschede, The Netherlands \\ ${ }^{2}$ Biomedical Signal and Systems, University of Twente, Enschede, The Netherlands \\ Email: gerjan.wolterink@utwente.nl
}

\begin{abstract}
Conductive 3D-printed structures made out of a carbon doped thermoplastic polyurethane (TPU) deposited by an FDM 3D-printer show a high inter-layer contact resistance. Due to this poor resistive coupling capacitive effects between layers become prominent. This effect can be used to create capacitive force sensors by depositing only two thin layers of material. In this paper we investigate the feasibility of such a 3D-printed force sensor. The change in capacitance due to the compression of the material caused by an applied force $(0 \mathrm{~N}$ to $10 \mathrm{~N})$ is measured using an LCR meter. The presented sensor concept has a high potential for implementation in biomedical and soft robotic applications since the sensor is thin and flexible because it is made from soft material.
\end{abstract} sensor

Keywords - 3D-Printing, Conductive, Flexible, Soft, Force

\section{INTRODUCTION}

Information about interaction forces between fingertips and the external environment is of great value in e.g. control of prosthesis, assistive devices and for the quantitative assessment of rehabilitation therapies. Nowadays such assessments are performed by clinicians or physical therapists but this could be well supported by thin force sensors placed at the fingertips. However, the study of Kortier et al. [1], showed that current force sensors have two main difficulties. Firstly, the sensors are usually made of stiff materials leading to loss of touch sensation by the user. Secondly, current sensors do not enable good sensor to skin attachment inducing large cross-sensitivity to unwanted movements caused by external forces [1].

3D-printed force sensors, fabricated using fused deposition modelling (FDM) technologies, could overcome these difficulties due to design freedom, customizability and availability of soft materials. Previously reported force sensors made using FDM printing technology are based on piezoresistive and capacitive principles [2]-[5]. The printed capacitive force sensor presented by Schouten et al. [3] consists out of two layers of conductive materials with several layers of an elastic dielectric material in between resulting in a thickness of $1.2 \mathrm{~mm}$. However, new insights in the anisotropy of conductive 3D-printed structures could lead to more flexible and thinner force sensors.

This works was developed within the SoftPro project, funded by the European Union's Horizon 2020 Research and Innovation Programme under Grant Agreement No. 688857

\section{TEChNOLOGY}

\section{A. Anisotropy in FDM printed objects}

Fused deposition modelling (FDM) is an additive manufacturing technique where a 3D-object is build up by melting and depositing a thermoplastic material. The raw materials are in the form of filament. Conductive materials are made by blending the thermoplastic host material with conductive particles such as copper or carbon [6]-[8]. During printing, the melted material is deposited in lines in the $x$ and $y$-directions by the printing head. To create height, the $z$-direction, layers of the material are stacked on to each other (Fig. 1). This leads to anisotropic conductive material due to the high interface resistance between the layers of the conductive material [9].
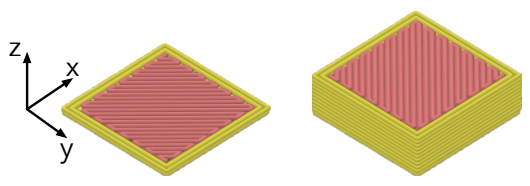

Fig. 1. Graphical illustration of the printing path in FDM 3D-printing.

Figure 2 shows an illustration of two structures with equal thickness. The right structure has twice as many layers as the left structure. This results in a higher impedance as measured over the $z$-direction caused by the increased number of interfaces within the material.

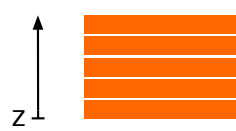

$\mathrm{R}$

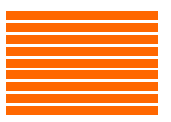

$2 \mathrm{R}$
Fig. 2. Graphical illustration of the influence of the interlayer resistance; doubling the number of layers will strongly increase the resistance as measured in the $z$-direction.

\section{B. Sensor principle}

Since the interlayer resistance is relatively high compared to the resistance of the material, it will constitute a capacitive contribution between layers. The general principle is based on the hypothesis that conductive particles are concentrated in the middle of the deposited material (see Fig. 3). When a flexible host material is used the distance between the layers will change when a force is applied, resulting in a change in capacitance. Using these materials force sensors can be made 
without the need to include extra layers of dielectric material. Therefore, force sensors can in principle be made by depositing only two thin layers of material, leading to flexible and thin structures.

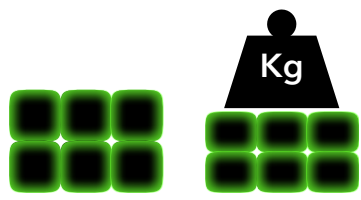

Fig. 3. Illustration of cross-section of two layers of material. Black represent concentration of carbon particles, green represent the dielectric material. Loading the structure leads to a shorter distance between the conductive cores and thus increasing capacitance.

\section{Methodology}

\section{A. Sensor fabrication}

Fig. 4 shows the design of a concept minimal sensor consisting of two partly overlapping layers (black parts) of conductive TPU (PI-ETPU, Palmiga Innovation, Sweden). As surrounding insulation material a non-conductive TPU (NinjaFlex, Fenner Drives, USA) is used. The designs are made using Fusion360 (Autodesk, USA). The selection of the infill pattern and printer control is handled by slicer software (Simplify3D, Inc., USA). The structures are printed on a consumer grade multi-material 3D-printer (Creator Pro, FlashForge Corporation, China) fitted with a dedicated extruder for flexible materials (Flexion Extruder,Diabase Engineering, USA).

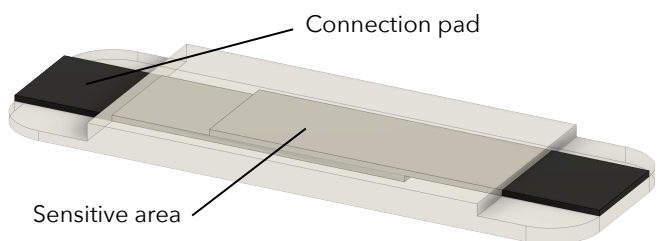

Fig. 4. CAD drawing of the concept sensor, black parts are conductive parts, gray parts are dielectric

\section{B. Measurement set-up}

The capacitance of the sensor is read out using an LCR meter (HP 4284A) in a 4-wire configuration. Therefore, four stranded wires are melted in the connection pads of the sensor. To ensure the whole structure is connected, the wires are connected over the whole width of the connection pad. These wires are as close as possible connected to the four shielded wires of the LCR meter. To prevent parasitic capacitance the shield of the measurement wires are connected to each other at the end and to the aluminium measurement set-up. The capacitance is read out at a frequency of $100 \mathrm{kHz}$ and a voltage of $1 \mathrm{~V}$.

The sensor is mounted on a vertical aluminium plate of the measurement set-up. A force-controlled linear actuator (SMAC LCA25-050-15F) in horizontal position is used to apply a force and read out the deflection of the sensor.

\section{Measurement protocol}

The actuation force of the linear actuator is controlled using a computer running Matlab (Mathworks Inc., USA). The actual force and position data from the linear actuator and the data from the LCR meter is recorded using the same Matlab program. To get insight in the response of the sensor, a load cycle alternating between $10 \mathrm{~N}$ and $0.1 \mathrm{~N}$ is applied to the sensor. Each cycle lasted $10 \mathrm{~s}$. The force capacitance relation is determined by applying a force ranging from $1 \mathrm{~N}$ to $10 \mathrm{~N}$ and back in increments of $1 \mathrm{~N}$. Each force increment lasted $5 \mathrm{~s}$. To get more detailed insights into the performance of lower forces, a second force range from $0.2 \mathrm{~N}$ to $1 \mathrm{~N}$ is applied in increments of $0.1 \mathrm{~N}$. Again each increment lasted $5 \mathrm{~s}$. The force capacitance relation is derived by taking the mean of the measured value of the force and capacitance over the $5 \mathrm{~s}$ increment windows.

\section{RESULTS}

To confirm the capacitive contribution to the impedance of stacked layers of doped TPU we first measured its impedance, using the set-up and method described above. Above $10 \mathrm{kHz}$ the structure shows a marked fall-off, see Fig. 5, indicative of such capacitive contributions.

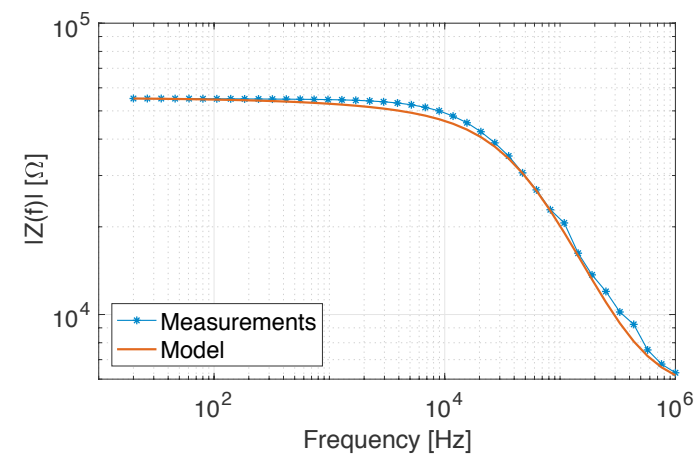

Fig. 5. Modulus of the impedance of a stack of 2 layers of carbon-doped TPU, clearly indicating the capacitive contribution.

Fig. 6 shows the relative change of the measured displacement and capacitance after an applied load ranging from $0 \mathrm{~N}$ to $10 \mathrm{~N}$. The force shows a clear square wave pattern, as programmed. The displacement and capacitance, however, show a damped behaviour, especially when the sensor is unloaded. The thermoplastic material of the sensor does not completely return to its original size within the $10 \mathrm{~s}$ of unloading. This effect is largely reflected in the measured capacitance and increases with the number of loadings, indicating the presence of creep in the stacked TPU material.

The relationship between the force and capacitance (Fig. 8) can be derived from the data shown in Fig. 7 by taking the mean and standard deviation of each $5 \mathrm{~s}$ interval. Fig. 7 shows that with increasing force the relative capacitance change decreases. It also shows that the relative capacitance change is more than 10 times larger than the relative resistance change. Fig. 9 shows the force capacitance relationship for forces up to $1 \mathrm{~N}$. The relatively large standard deviation for forces between $0.2 \mathrm{~N}$ to $0.4 \mathrm{~N}$ is caused by inaccuracies of the linear actuator. 


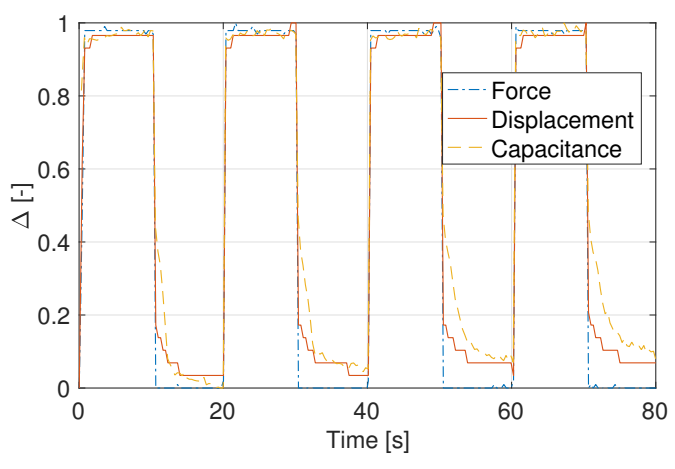

Fig. 6. Relative change of the applied force and measured displacement and capacitance. The applied force was a square wave ranging from $0 \mathrm{~N}$ to $10 \mathrm{~N}$.
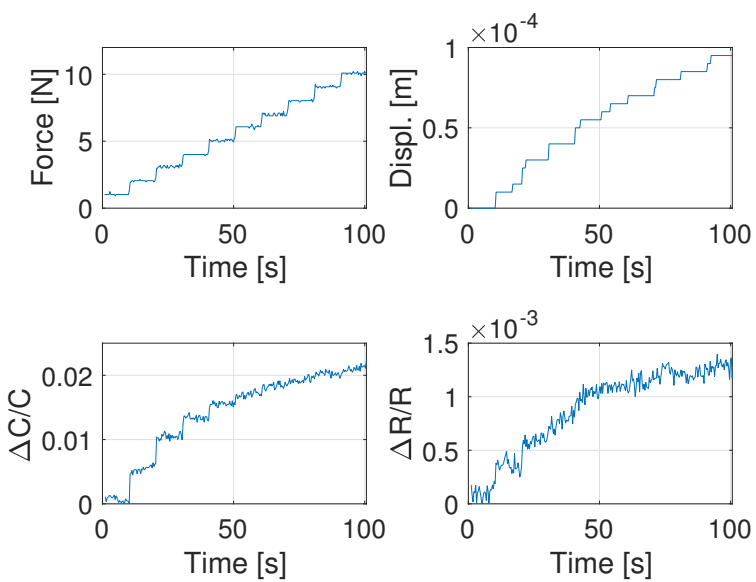

Fig. 7. Raw data for a force increasing from $1 \mathrm{~N}$ to $10 \mathrm{~N}$ in increments of $1 \mathrm{~N}$. Left top shows the applied force, right top the measured deflection, left bottom the measured relative capacitance change, right bottom the measured relative resistance change.

Fig. 10 shows the relative capacitance change against force obtained from 10 load cycles of $10 \mathrm{~N}$ over time. The figure shows a considerable amount of hysteresis. However, no clear drift relationship over time is observed.

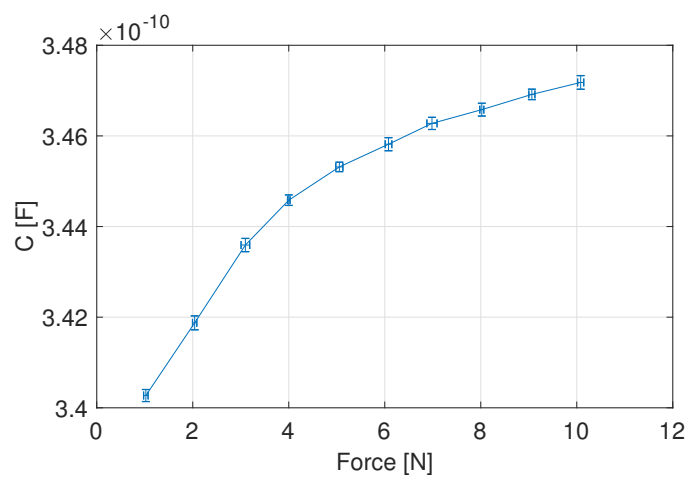

Fig. 8. Mean capacitance and mean force including standard deviations derived from Fig. 7

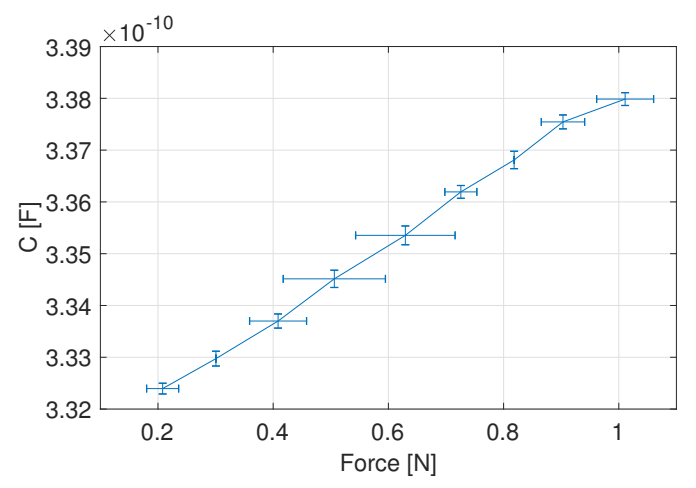

Fig. 9. Mean capacitance and mean force including standard deviations for low forces.

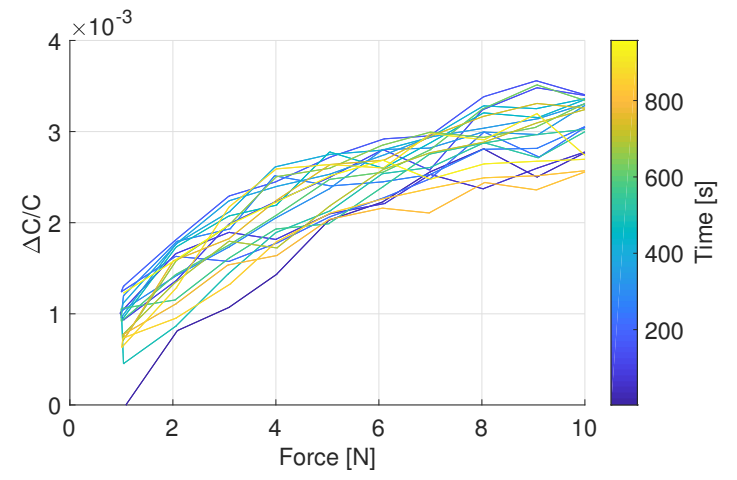

Fig. 10. Relative capacitance change over 10 cycles of $10 \mathrm{~N}$ over time.

\section{DISCUSSION}

Analysis of the printed sensor shows a non-linear capacitance force behaviour, the sensor shows a bigger capacitance change for lower forces than for higher forces. This effect is likely caused by the properties of the used thermoplastic elastomer. Other effects of the material, such a dampening and creep are also present and visualized by Fig. 6 .

The presented sensor uses the anisotropic properties of the printed material. At the moment only the high inter-layer contact resistance in the $z$-direction is used. However, this high contact resistance is also present in the $x$ and $y$-directions. These structures are more complex since they are in most cases lines in a meandering pattern. Future improvements can be made by further exploiting the material properties and the influence of printing parameters, such as the extrusion speed and temperature, since these may be determining the (re)distribution of carbon in the printed track elements ('traxels')

\section{CONCLUSIONS}

We have shown a working concept of a thin, flexible 3D-Printed capacitive force sensor that uses the anisotropic properties of an FDM 3D-printed structure. This allows the fabrication of capacitive force sensors by only depositing two layers of material. Therefore, the sensor maintains its soft and flexible properties making it useful for biomedical and soft robotic applications. Further improvements to the sensor are to be made by gaining further understanding of the influence of different design choices of the sensor and fully exploiting the extrusion process. 


\section{REFERENCES}

[1] H. Kortier, "Assessment of hand kinematics and interactions with the environment," University of Twente, February 2018.

[2] E. Perez, "3d printed sensor to detect muscle contraction by means of force myography," Master's thesis, University of Twente, November 2017.

[3] M. Schouten, R. Sanders, and G. Krijnen, "3D printed flexible capacitive force sensor with a simple micro-controller based readout," in 2017 IEEE SENSORS, Oct 2017, pp. 1-3.

[4] S. J. Leigh, R. J. Bradley, C. P. Purssell, D. R. Billson, and D. A. Hutchins, "A simple, low-cost conductive composite material for $3 \mathrm{~d}$ printing of electronic sensors," PloS one, vol. 7, no. 11, p. e49365, 2012.

[5] M. B. Kirkpatrick, J. A. Tarbutton, T. Le, and C. Lee, "Characterization of $3 \mathrm{~d}$ printed piezoelectric sensors: Determiniation of $\mathrm{d} 33$ piezoelectric coefficient for 3d printed polyvinylidene fluoride sensors," in SENSORS, 2016 IEEE. IEEE, 2016, pp. 1-3.

[6] Multi3D. Electrifi Conductive 3D Printing Filament. Https://www.multi3dllc.com/product/electrifi-3d-printing-filament/, Accessed: 13-06-2017. [Online]. Available: https://www.multi3dllc.com/ product/electrifi-3d-printing-filament/

[7] ProtoPlant, makers of Proto-pasta. Composite PLA - Electrically Conductive Graphite. Url: https://www.proto-pasta.com/, Accessed 31-01-2017. [Online]. Available: https://www.proto-pasta.com/

[8] Palmiga Innovation. Material info for PI-ETPU 95-250 Carbon Black the conductive and flexible 3D printing filament. Url: http://rubber3dprinting.com/pi-etpu-95-250-carbon-black/, Accessed: 26-05-2017. [Online]. Available: http://rubber3dprinting.com/ pi-etpu-95-250-carbon-black/

[9] B. Eijking, R. Sanders, and G. Krijnen, "Development of whisker inspired 3d multi-material printed flexible tactile sensors," in SENSORS, 2017 IEEE. IEEE, 2017, pp. 1-3. 